\title{
Typologie des procédés scripturaux des salons de clavardage en français chez les adolescents et les adultes
}

\author{
Anaïs Tatossian \\ Université de Montréal \\ anais.tatossian@gmail.com
}

\section{Introduction}

Le développement des nouvelles technologies a donné naissance à de nouvelles formes de communication écrite, dite « communication médiée par ordinateur » (CMO), aujourd'hui devenue incontournable dans presque toutes les sphères de l'activité humaine. Celle-ci se présente sous une diversité de formes qu'on peut classer en deux groupes selon la temporalité de l'échange :

1) la communication en temps réel, soit synchrone quand le message est reçu aussitôt qu'il est envoyé par l'émetteur (par ex. le clavardage ${ }^{1}$, la messagerie instantanée);

2) la communication en différé, soit asynchrone lorsque survient un décalage temporel entre l'émission et la réception du message (par ex. le courrier électronique, les forums de discussion).

Tant synchrone qu'asynchrone, la CMO a fait émerger des stratégies d'écriture nouvelles. La prise en compte de ce paramètre nous semble indispensable dans la mesure où la temporalité de l'échange conditionne en partie du moins certaines manifestations linguistiques et discursives de ces modes d'échange.

L'objet de notre étude est le dispositif de conversation en groupe IRC (Internet Relay Chat), qui présente deux caractéristiques importantes aux plans discursif et communicationnel. D'une part, le clavardage est une forme de communication hybride : le code utilisé est l'écrit, mais les échanges de messages entrent dans une structure de dialogue, rappelant l'oral spontané (cf. Luzatti 1991; Jeay 1991; Werry 1996; Anis 1999; Crystal 2006). Il s'agit d'une conversation sous forme écrite, en temps réel, impliquant un ou plusieurs participants en simultané. D'autre part, la rapidité des échanges est cruciale tant pour le décodage que pour l'encodage des messages : les temps de réaction disponibles sont courts et la célérité de l'expression est requise pour soutenir le fil de la conversation.

Les divers dispositifs de CMO ont conduit à l'émergence de stratégies scripturales nouvelles dont la variabilité dépend crucialement de la dimension synchrone ou asynchrone des échanges. Les études sur le clavardage s'entendent (voir notamment Werry 1996; Anis 1999; Pierozak 2000) sur l'importance de deux facteurs : le temps, qui incite les clavardeurs à recourir à des stratégies d'abréviation et l'emploi de marques d'expressivité qui ont pour effet de réduire l'écart entre l'oral et l'écrit. Étant donné que le clavardage consiste à établir une communication en temps réel fondée uniquement sur le texte, sans transmettre l'image ou la voix des scripteurs ${ }^{2}$ (absence de canal sensoriel visuel et auditif), les clavardeurs sont tentés de reproduire les paramètres de la conversation en face-à-face: expressions faciales, intonations, pauses, expression des émotions, etc.

Les adolescents, avides d'identité, sont exposés précocement aux nouveaux usages communicationnels, et d'autant plus que l'écriture électronique constitue un moyen de résister aux contraintes imposées par le monde des adultes (Sebba 2003). Selon Gadet, des usages scripturaux apparaissent chez les adolescents, «puisque conformes à leur désir de bousculer les conventions du standard » (citée dans Anis $2001: 62$ ). Il se dégage un réel désir de démarcation entre les jeunes et les adultes. Notre étude s'inscrit dans cette réflexion puisqu'il s'agit d'une étude comparative entre les usages scripturaux d'adolescents par contraste avec ceux d'adultes, étant entendu que les scripteurs adultes n'échappent pas non plus à la contrainte temporelle de la dynamique du clavardage. Indépendamment de la question identitaire, les deux groupes 
disposent en effet de ressources similaires en termes matériels et quel que soit l'âge du clavardeur, chacun doit soutenir le rythme des échanges.

\section{2 État de la recherche sur la CMO en mode synchrone}

La notion de Computer-Mediated Communication (CMC) est apparue dans le milieu anglo-saxon au début des années quatre-vingts pour décrire l'émergence d'un nouvel usage communicationnel de l'ordinateur. L'introduction de ce concept s'accompagnait d'une tendance à reconsidérer l'ordinateur, «médium» plutôt qu' «outil» servant à effectuer des calculs. Pour illustrer ce changement de perspective, il convient de reprendre la définition de la CMC proposée par Herring (2000) : «Computermediated communication: Human-human communication by means of messages transmitted via computer networks ». Il est donc question d'un dialogue personne-personne par ordinateur, ou, autrement dit, d'une interaction humain-machine-humain.

La recherche sur la CMO s'est constituée au tournant des années quatre-vingts selon une perspective psychosociologique, avec l'étude de Kiesler, Siegel et McGuire (1984) sur la comparaison entre les processus de communication en face-à-face et la CMO. Les stratégies communicationnelles qui découlent de la CMO suscitent dès lors l'intérêt des sciences humaines : sociologues, psychologues, spécialistes des sciences de la communication les ont tour à tour analysées. Ces études ont permis notamment d'explorer les questions relatives à la création de liens sociaux, à celle de l'identité, au développement d'une communauté, toujours mises en rapport avec les techniques de la communication.

En linguistique, un domaine fécond et encore peu exploré s'ouvre à l'analyse. Les premières études sur les échanges synchrones sont apparues il y a une quinzaine d'années et portaient sur des textes produits par l'intermédiaire du Minitel (Jeay 1991; Luzzati 1991). C'est au cours des dix dernières années, grâce aux travaux sur le clavardage de Werry (1996) sur l'anglais, de Anis (1999) et de Pierozak (2001) sur le français, de Llisterri (2002) sur l'espagnol, et de Zelenkauskaite et Herring (2006) sur le lituanien et le serbo-croate, que s'est développé un courant de recherche à proprement parler. Mentionnons aussi l'enquête de Lacerte (2006) à visée psychosociologique sur les usages et les représentations sociales de la qualité de la langue française chez les jeunes clavardeurs. Les seules études recensées sur la messagerie instantanée sont celles de Palfreyman et Al Khalil (2003) sur l'arabe et de Tagliamonte et Denis (2006) sur l'anglais.

Bien que le clavardage transite par un clavier, il est donc «écrit», des chercheurs ont constaté que ce type de communication électronique ne correspond univoquement ni à l'écrit, ni à l'oral. Les études sur le sujet mettent en évidence le rapprochement entre l'oral spontané et l'écrit parce que «bon nombre de ces énoncés sont conçus dans le fil de leur énonciation, ce qui est la principale caractéristique de l'oral spontané » (Luzatti 1991 : 101). Jeay (1991) nomme cette forme de communication un "parlécrit», Werry (1996) suggère l'appellation «interactive written discourse », Anis (1999 : 74) propose la dénomination de "conversation écrite» et, plus récemment, Crystal (2001) suggère le terme «Netspeak». Dans le cadre de notre recherche, nous adoptons le terme de «scripto-clavardage » qui couvre tous les usages scripturaux dans une activité bien circonscrite à l'intérieur du « Netspeak».

Notre étude propose une analyse des ressources scripturales mises au point par les utilisateurs de forums de clavardage de langue française. Si cette forme de communication écrite se rattache en partie à l'oral spontané, nous pouvons émettre l'hypothèse que

les usages graphiques employés en français en situation de clavardage (système de discussion en mode synchrone) s'écartent au moins en partie de l'orthographe conventionnelle.

Nous avons voulu répondre à deux questions, soit :

- de quelle manière peut-on classifier les usages scripturaux attestés ?

- ces usages graphiques sont-ils les mêmes chez les adultes et les adolescents aux plans qualitatif et quantitatif? 
Nous présenterons dans la suite les données et la méthodologie utilisée $(\S 3)$, notre typologie $(\S 4)$ et finalement une comparaison entre les usages des adolescents et des adultes $(\S 5)$.

\section{Données et méthodologie}

Notre étude se fonde sur un corpus d'échanges effectués au moyen du protocole Internet Relay Chat (IRC), qui se déroulent en temps réel (mode synchrone). Les logiciels clients IRC ont des fonctions qui permettent d'enregistrer les conversations, publiques ou privées, dans un fichier log (fichier texte). Une fois enregistré sur un support de mémoire, le fichier $\log$ se prête à toute analyse sans nécessiter la connexion au serveur IRC. La Figure 1 illustre un exemple d'échange extrait d'un canal nommé \#50ans\&+ dans lequel on distingue les messages émis par les clavardeurs des commandes générées par le serveur IRC (lignes commençant par un astérisque).

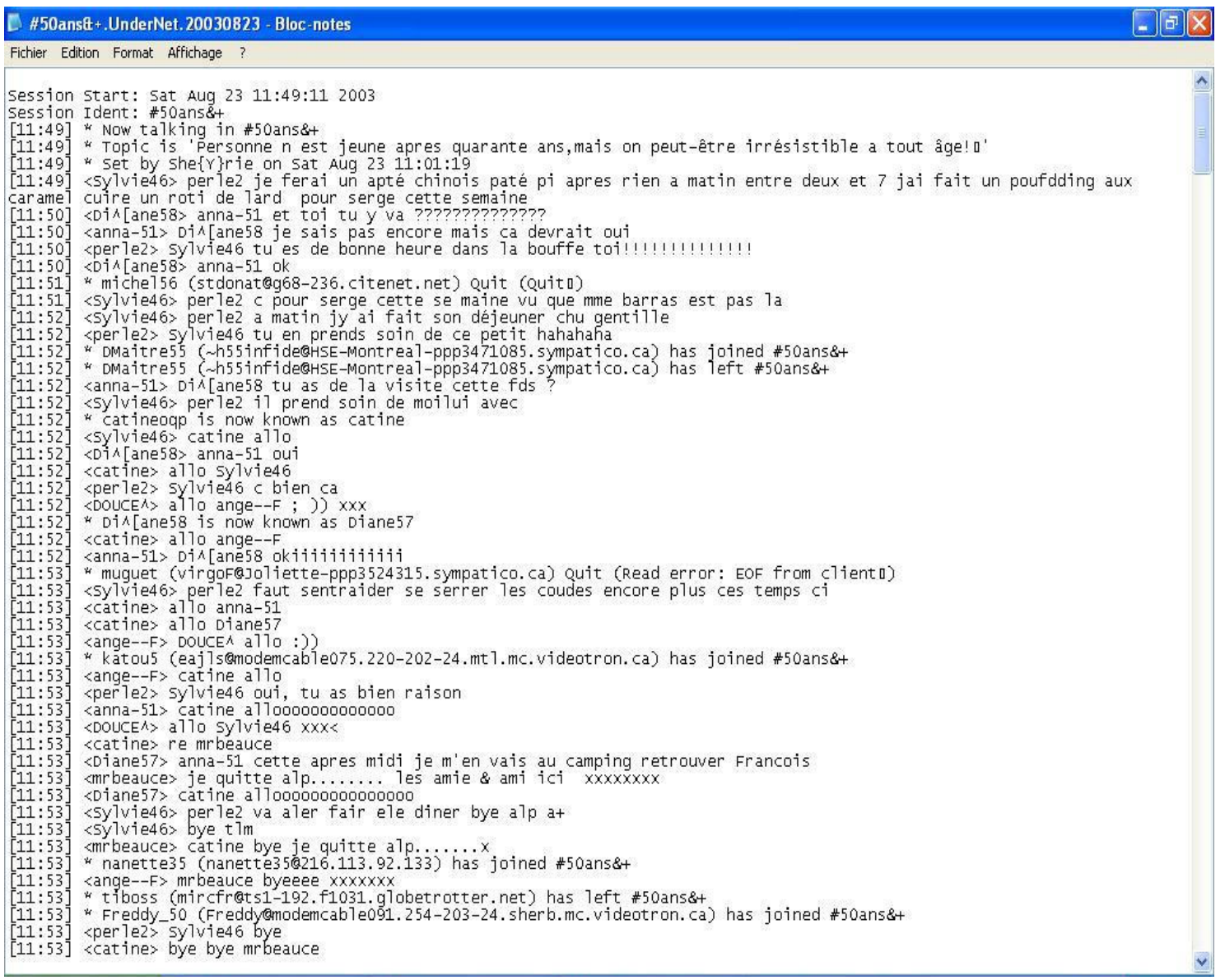

Figure 1 - Saisie d'écran d'un fichier log : extrait du canal \#50ans\&+

Nous avons analysé un corpus constitué de 4520 messages $^{3}$ échangés sur différents canaux IRC provenant du réseau Undernet ${ }^{4}$ (vingt-deux sessions) dont 2260 proviennent de canaux pour adultes et 2260 de canaux pour adolescents 5 . Le corpus est constitué d'échantillons sur des canaux francophones québécois. Nous avons enregistré les conversations en tant qu'observateur neutre, c'est-à-dire sans envoyer de messages publics ou privés afin de ne pas influencer les échanges en cours. 
Pour identifier les différents messages, nous avons numéroté les énoncés en commençant par le numéro 1 dans chacun des canaux. Les lettres $\mathrm{A}$ (adulte) et $\mathrm{J}$ (jeune) indiquent que les messages sont tirés respectivement du canal pour adultes et de celui pour adolescents. Par exemple, le message suivant est tiré du corpus A :

(2) A I $129<$ sylvy_^> police40 ici sa tombe pas mal avec orage

\section{Typologie des procédés scripturaux dans les salons de clavardage en français}

Dans cette section, nous présentons notre modèle de classification mis au point sur la base des phénomènes scripturaux recensés. Nous avons travaillé avec quatre catégories générales qui rendent compte de toutes les variantes graphiques et scripturales de notre corpus, étant entendu que chacune comporte plusieurs sous-catégories : les procédés abréviatifs, les substitutions de graphèmes, les neutralisations en finale absolue et les procédés expressifs. Le Diagramme 1 fait la synthèse de notre typologie. 
ISBN 978-2-7598-0358-3, Paris, 2008, Institut de Linguistique Française

Sociolinguistique et écologie des langues DOI $10.1051 / \mathrm{cmlf0} 08012$
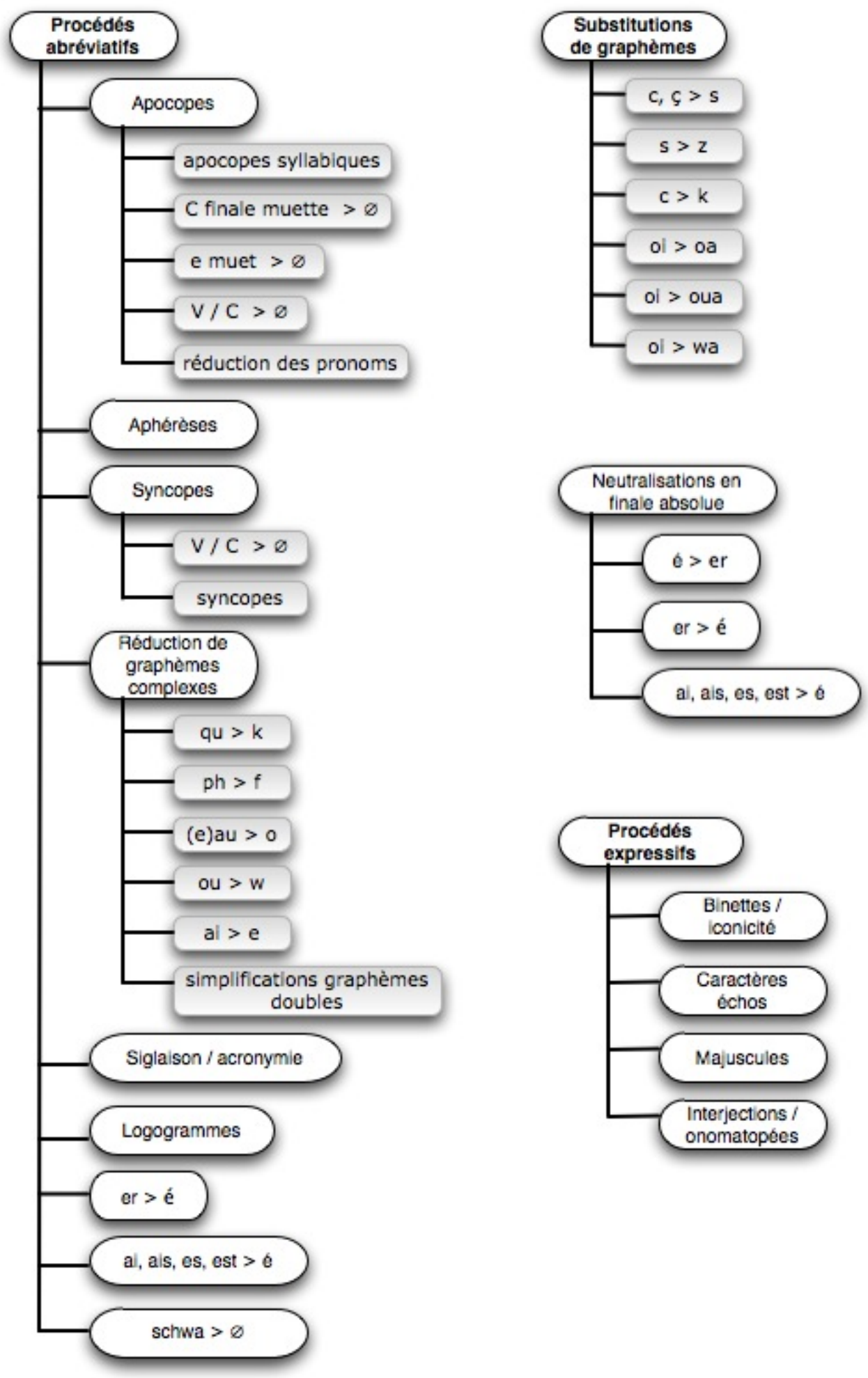

Diagramme 1 - Typologie des procédés scripturaux en situation de clavardage 


\subsection{Les procédés abréviatifs}

Frei (1929: 106) a souligné le besoin d'économie comme un «facteur indéniable dans la vie du langage », et dans lequel on " cherche à abréger autant que possible la longueur et le nombre des éléments dont l'agencement forme la chaîne parlée ». On pourrait transposer cette recherche de brièveté au clavardage, dont l'une des caractéristiques principales repose sur l'extrême rapidité des échanges. L'objectif est en conséquence de réduire le temps de production des messages à l'aide de stratégies diverses dont nous faisons ci-dessous un survol. On peut répartir les différentes simplifications orthographiques qui en découlent dans dix sous-catégories.

1) Les apocopes : elles correspondent à une réduction de la forme qui opère à la finale de l'unité. Nous reconnaissons cinq cas de figure :

i. les apocopes syllabiques, par ex., avec maintien ici du $s$ pluriel :

- A I $422<$ gerard3 > on peux tu faire des dessins ici les ops <opérateurs>;

ii. la chute de la consonne finale muette, par ex. :

- J II $132<\{\mathrm{M}\{\mathrm{eL}\} \mathrm{i}\}>$ sérieu? < sérieux>;

iii. la chute du $e$ muet à la finale, par ex. :

- J II $483<$ EppoKKK $>$ ta gueul < gueule>;

iv. la chute de voyelles et/ou de consonnes, par ex. :

- J VI $11<$ StepH_> ya tu d < des $>$ gars qui veulent parler ak une fille de 14 ans?

v. la réduction des pronoms, par ex. :

- A I $257<$ SaraAway $>$ jreviens $<$ je reviens $>$ demain :).

2) Les aphérèses : elles concernent un «changement phonétique qui consiste en la chute d'un phonème initial ou en la suppression de la partie initiale d'un mot» (J. Dubois et al. 2001 : 43), par ex. autobus devient bus. L'exemple suivant tiré de notre corpus illustre cette catégorie :

- J VI $204<$ g_be $>$ ki a une cam <webcam $>$ ?

3) Les syncopes : elles correspondent à la «disparition d'un ou plusieurs phonèmes à l'intérieur d'un mot » (Dubois et al. 2001 : 464). Il s'agit pour nous de la suppression de certains graphèmes à l'intérieur du mot, par ex. : $t$ t pour tout. Pour rendre compte assez finement de nos données, nous avons réparti les syncopes en deux sous-catégories. Nous distinguons ainsi d'une part, celles qui créent des suites de graphèmes dont la lecture, soit l'oralisation, restitue la forme phonétique du mot, d'autre part, les syncopes qui n'ont pas cet effet. Par exemple, nous contrastons « dcd »<décédé $>$ de «bcp » $<$ beaucoup $>$. La forme «dcd», lue, restitue [desede], ce qui n'est pas le cas de «bcp»* [besepe]. Le premier cas s'apparente au mode siglaison ${ }^{6}$, le second à la sténographie, ou à la prise de notes.

4) La réduction de digrammes et de trigrammes : elle concerne la réduction d'un groupe de deux (digramme) ou de trois graphèmes (trigramme) à un seul graphème. Nous avons relevé six cas :

i. $q u>k$, par ex. :

- J XI $72<$ D[er]EsSe $>$ mon chien vient dembarker $<$ d'embarquer $>$ sul clavier :P;

ii. $\mathrm{ph}>\mathrm{f}$, par ex. :

- A V $176<$ alyyy-h> femXLVIII j'aimerais te montrer une foto <photo $>$ que g pris cette après midi;

iii. (e)au > o, par ex. : 
- A XVII 65 <pasmoi> hehe, ok, c'est vrai, si vous aviez un escabo <escabeau> avec vous :);

iv. ou $>$ w, par ex. :

- A XIV $<$ Amy $\mid$ Ly $>$ win $<$ ouin $>$ mal a tete mais sa va;

v. ai $>$ e, par ex. :

- J II $496<\mathrm{MeLi}^{\wedge}$ Jre $>$ sexy j'tle lesse <laisse>;

vi. simplification des graphèmes doubles, par ex. :

- J III $14<[\mathrm{Gr} \mid$ ng0 $]>$ apres sa c corect $<$ correct $>=$ );

5) La siglaison concerne la formation d'un sigle, c'est-à-dire la «lettre initiale ou le groupe de lettres initiales constituant l'abréviation de certains mots [...]» (Dubois et al. 2001 : 429), par ex. :

- A VII $451<$ MimieQc $>$ allo tlm $<$ tout le monde $>$ :))).

6) L'acronymie relève de la siglaison. Il s'agit d'un sigle « prononcé comme un mot ordinaire » (Dubois et al. $2001: 13$ ), par ex. :

- A I $10<$ GRIZZLY-> miNoU^ lol <laughing out loud>.

7) Les logogrammes sont des «graphèmes qui correspondent directement à des morphèmes » (Anis 1999 : 77). Il s'agit de la substitution d'une unité lexicale par un symbole, par ex. :

- J VI $129<$ macy_love> bon ben bye tlm je vais revenir tanto moi la...a+<à plus> xxxxxx.

8) La neutralisation de la finale $\boldsymbol{e r}$ en $\boldsymbol{e}$ (er > é) (cf. § 4.3 pour explication et exemple);

9) La neutralisation des graphèmes complexes ai, ais, es, est en $\dot{\boldsymbol{e}}$ à la finale absolue (ai, ais, es, est $>$ é) (cf. § 4.3 pour explication et exemple);

10) L'effacement du schwa à l'intérieur du mot (les effacements à la finale du mot sont rangés avec les apocopes), par ex. :

- A XVII $55<$ ladylight> Shysky21 surment <sûrement> mais connais pas tous le monde désoler.

\subsection{Les substitutions de graphèmes}

Notre corpus comporte de nombreuses variantes autres qu'abréviatives. Il s'agit de substitutions de graphèmes. Cette opération consiste à remplacer un graphème par un autre dans le but de transcrire l'oral (phonétisation). Par ex., la substitution du graphème $\mathbf{c}$ par $\mathbf{k}$ dans cabane produit kabane. Nous avons recensé six cas de figure dans notre corpus :

1) c, ç > s, par ex. :

- J VI $2<$ Kamahl $>$ hmmm sa $<$ ça $>$ peut etre interessant;

2) $s>z$, par ex. :

- J II $522<\mathrm{M} 4 \mathrm{G}>$ tout un plaizir $<$ plaisir $>$ math;

3) c $>$ k, par ex. :

- A I $58<$ miNoU$^{\wedge}>$ pleinpsou té rekonnu $<$ reconnu $>$ le voizin :);

4) oi $>$ oa, par ex. :

- J XXI $57<$ marieecko> ya tu kk1 ki veut parler ak moa <moi> ?; 
5) oi > oua, par ex. :

- J VI $475<$ F0X-GiRl $>$ t en math koua $<$ quoi $>$;

6) oi $>$ wa, par ex. :

- A XVI $41<\{\mathrm{L} \mid \mathrm{sE}\}>$ wZoe heu ta perdu ta langue twa $<$ toi $>$ ?????.

Contrairement aux procédés abréviatifs, les remplacements de graphèmes ne visent pas l'économie, mais constituent strictement une tentative de reproduire l'oral à l'aide d'interventions de type phonéticographiques.

\subsection{Les neutralisations en finale absolue}

Les neutralisations en finale absolue semblent traduire une gestion cognitive laxiste du code écrit, sans doute pour des raisons qui tiennent à la situation de production (pression du temps, rapidité). Nous avons rencontré trois situations :

1) é > er, par ex. :

- A I $77<$ pic666> wowowowowo je suis habiller <habillé> la;

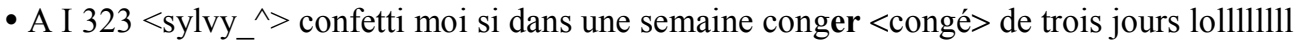

2) er $>$ é, par ex. :

- A VII $155<$ Lunedor42> Laidpreux il est fru après moi parce que j ai pas voulu le rencontré $<$ rencontrer $>$ le pov tit pet loll;

3) ai, ais, es, est > é, par ex. :

- J XIII $52<$ RoXie_> elle veut maigrir dé $<$ des $>$ doigt.

Les cas 2) et 3) se retrouvent aussi dans la catégorie des procédés abréviatifs en raison de l'économie de caractères (un caractère pour deux ou trois caractères). Le cas 1) est manifestement une substitution de luxe (deux caractères pour un seul) mais elle permet dans certains cas l'économie de gérer les règles d'accord complexes du participe passé.

\subsection{Les procédés expressifs}

La propension à l'expressivité dans les forums de clavardage rappelle sans nul doute la spontanéité de l'oral; elle se manifeste par la simulation des paramètres de la conversation en face-à-face. Nous avons recensé quatre « manipulations » propres à simuler une situation d'échange oral.

1) Les binettes (smileys) : elles sont composées de caractères ASCII et traduisent une émotion, par ex., la binette «:-( » (les deux-points suivis d'un tiret puis d'une parenthèse ouvrante) indique le mécontentement.

2) Les caractères écho : il s'agit de la répétition de caractères dans le but de simuler l'accent d'insistance (Léon 1992 : 26); ils enrichissent l'échange par ajout d'une information para-linguistique, par ex. :

- A I $13<$ GRIZZLY-> sylvy_^ riennnnnnnnnnnn mais rien lollllllll et toi.

3) Les caractères en majuscules : ils reflètent l'exacerbation d'une émotion - négative comme un sentiment de colère, ou positive comme la joie - et donnent l'impression que le clavardeur produit son message en criant, par ex. :

- J II $283<\{\mathrm{M}\{\mathrm{eL}\} \mathrm{i}\}>$ AH NON PAS MATH!;

- J X $27<\mid$ ei|a> LACHE SURTOUT PAS!. 
4) Les interjections/onomatopées : les clavardeurs en usent pour simuler le contact à la fois visuel et auditif de la conversation de visu, pour marquer les pauses, les hésitations, ou encore pour reproduire certains bruits, par ex. :

\section{- J VI $490<$ F0X-GiRl $>$ :( snif sniff.}

Soulignons qu'elles se combinent souvent avec les caractères écho, par ex. :

- A IV $86<$ marie27> Voltair26 wooooooooo la lache hell_man grrrrrrrrrr.

\section{Analyse des résultats}

À partir de notre modèle de classification des procédés graphiques, nous avons vérifié si nos deux populations recourent proportionnellement aux mêmes moyens scripturaux. Le Tableau 1 donne les résultats quantifiés pour les cinq grandes catégories établies en distinguant nos groupes : jeunes (J) d'une part, adultes (A) de l'autre.

\begin{tabular}{|c|c|c|c|c|c|}
\hline \multirow[t]{2}{*}{ Catégories } & \multirow[t]{2}{*}{ Exemples } & \multicolumn{2}{|c|}{$\mathbf{J}$} & \multicolumn{2}{|c|}{$\mathbf{A}$} \\
\hline & & $\begin{array}{l}\text { Nb } \\
\text { occ. }\end{array}$ & $\%$ & $\begin{array}{l}\mathrm{Nb} \\
\text { occ. }\end{array}$ & $\%$ \\
\hline $\begin{array}{l}\text { Procédés } \\
\text { abréviatifs }\end{array}$ & $\begin{array}{l}\text { (e)au }>\text { o } \\
\text { A I } 47<\text { pleinpsou }>\text { moi en bateau } \\
\text { sylvy_^ et il etais tres bo le ciel } \\
<\text { bo : beau }>\end{array}$ & 1675 & $65,8 \%$ & 1152 & $52,7 \%$ \\
\hline $\begin{array}{l}\text { Substitutions de } \\
\text { graphèmes }\end{array}$ & $\begin{array}{l}\mathrm{s}>\mathrm{z} \\
\text { A I } 58 \mathrm{miNoU}^{\wedge}>\text { pleinpsou té rekonnu } \\
\text { le voizin :) } \\
<\text { voizin : voisin }>\end{array}$ & 277 & $10,9 \%$ & 97 & $4,4 \%$ \\
\hline $\begin{array}{l}\text { Neutralisations en } \\
\text { finale absolue }\end{array}$ & $\begin{array}{l}\text { é }>\text { er } \\
\text { A XVI } 46<\text { rock } 57>\text { tu } \mathrm{m} \text { as encore } \\
\text { oublier } \\
\text { <oublier: oublié> }\end{array}$ & 153 & $6,0 \%$ & 104 & $4,8 \%$ \\
\hline $\begin{array}{l}\text { Procédés } \\
\text { expressifs }\end{array}$ & $\begin{array}{l}\text { caractères écho } \\
\text { A I } 13<\text { GRIZZLY-> sylvy_^ } \\
\text { riennnnnnnnnnnn mais rien lollllllll et } \\
\text { toi } \\
<\text { lol : laughing out loud }>\end{array}$ & 441 & $17,3 \%$ & 833 & $38,1 \%$ \\
\hline & $\sum$ & 2546 & $100 \%$ & 2186 & $100 \%$ \\
\hline
\end{tabular}

Tableau 1 - Nombre d'occurrences et pourcentage pour chacune des catégories chez les adolescents et les adultes 


\subsection{Résultats détaillés}

\subsubsection{Les procédés abréviatifs}

Les procédés abréviatifs dominent dans les deux groupes et de façon encore plus nette chez les adolescents où ils représentent un peu plus de $65 \%$ de tous les procédés. Nous présentons dans le Tableau 2 les résultats pour chacune des sous-catégories.

\begin{tabular}{|l||r|r|r|r|}
\hline \multicolumn{1}{|c||}{ Procédés abréviatifs } & \multicolumn{2}{c||}{ J } & \multicolumn{2}{c|}{ A } \\
\hline & $\begin{array}{c}\text { Nb } \\
\text { Occ. }\end{array}$ & \multicolumn{1}{c|}{$\%$} & \multicolumn{1}{c|}{$\begin{array}{c}\text { Nb } \\
\text { occ. }\end{array}$} & \multicolumn{1}{c|}{$\%$} \\
\hline Apocopes & 752 & $44,9 \%$ & 349 & $30,3 \%$ \\
\hline $\begin{array}{l}\text { Siglaison/ } \\
\text { acronymie }\end{array}$ & 335 & $20,0 \%$ & 482 & $41,8 \%$ \\
\hline $\begin{array}{l}\text { Réductions de } \\
\text { digrammes et de } \\
\text { trigrammes }\end{array}$ & 283 & $16,9 \%$ & 106 & $9,2 \%$ \\
\hline Syncopes & 107 & $6,4 \%$ & 72 & $6,3 \%$ \\
\hline ai, ais, es, est >é & 94 & $5,6 \%$ & 60 & $5,2 \%$ \\
\hline Aphérèses & 43 & $2,6 \%$ & 46 & $4,0 \%$ \\
\hline Logogrammes & 33 & $2,0 \%$ & 21 & $1,8 \%$ \\
\hline Schwa > $\varnothing$ & 19 & $1,1 \%$ & 8 & $0,7 \%$ \\
\hline er > é & 9 & $0,5 \%$ & 8 & $0,7 \%$ \\
\hline \hline & 1675 & $100 \%$ & 1152 & $100 \%$ \\
\hline
\end{tabular}

Tableau 2 - Nombre d'occurrences et pourcentage des procédés abréviatifs chez les adolescents et les adultes

Les jeunes affectionnent clairement les apocopes, qui comptent pour près de $45 \%$ de tous les procédés abréviatifs mis en œuvre (752 occurrences). Au deuxième rang, on trouve la siglaison/acronymie qui, avec 335 occurrences, compte pour $20 \%$ des procédés abréviatifs. À elles seules, ces deux manipulations comptent ainsi pour $65 \%$ des procédés abréviatifs chez les adolescents. Au troisième rang, vient la souscatégorie de la réduction de digrammes et de trigrammes, qui compte pour près de $17 \%$ des techniques d'abréviation (283 occurrences). Les six sous-catégories restantes, syncopes (107 occurrences, soit 6,4 $\%$ ), neutralisation des graphèmes complexes ai, ais, es, est en é à la finale absolue (94 occurrences, soit $5,6 \%$ ), aphérèses (43 occurrences, soit 2,6\%), logogrammes (33 occurrences, soit 2,0\%), effacement du schwa (19 occurrences, soit $1,1 \%$ ), et neutralisation de la finale er en é ( 9 occurrences, soit $0,5 \%$ ) comptent pour un maigre cinquième du total de l'ensemble. L'abondance des procédés abréviatifs corrobore les observations d'il y a presqu'un quart de siècle de Walter (1984) sur le français branché de l'époque, dans lequel l'abréviation est responsable d'un grand nombre d'innovations lexicales.

Chez les adultes, les sigles/acronymes occupent la première position des procédés abréviatifs; ils représentent près de $42 \%$ des abréviations (482 occurrences). Les apocopes occupent la deuxième position, totalisant près du tiers de ces procédés (349 occurrences), les réductions de digrammes et de trigrammes la troisième position (106 occurrences, soit 9,2\%). Viennent ensuite les syncopes (72 occurrences, soit $6,3 \%$ ), les neutralisations des graphèmes complexes ai, ais, es, est en é à la finale absolue (60 occurrences, soit 5,2\%), les aphérèses (46 occurrences, soit 4,0\%), les logogrammes (21 
occurrences, soit 1,8\%), l'effacement du schwa ( 8 occurrences, soit $0,7 \%$ ) et la neutralisation de la finale er en é ( 8 occurrences, soit $0,7 \%$ ). Comme chez les adolescents, les six dernières sous-catégories totalisent ensemble près du cinquième de tous les procédés abréviatifs.

\subsubsection{Les procédés expressifs}

Dans les deux groupes, les procédés expressifs occupent le deuxième rang : 441 occurrences, soit 17,3\% chez les jeunes et 833 occurrences, soit $38,1 \%$ chez les adultes. Cette tendance à l'expressivité mime sans nul doute la spontanéité de l'oral. Le Tableau 3 recense les résultats obtenus pour les différentes sous-catégories. Signalons qu'en raison de la surabondance des binettes dans notre corpus, nous avons renoncé à les compter, de sorte que nous chiffrons seulement trois des quatre procédés de la catégorie.

\begin{tabular}{|l||r|r||r|r|}
\hline \multicolumn{1}{|c||}{ Procédés expressifs } & \multicolumn{2}{c||}{ J } & \multicolumn{2}{c|}{ A } \\
\hline & $\begin{array}{c}\text { Nb } \\
\text { occ. }\end{array}$ & \multicolumn{1}{c|}{$\%$} & $\begin{array}{c}\text { Nb } \\
\text { occ. }\end{array}$ & \multicolumn{1}{c|}{$\%$} \\
\hline Caractères écho & 241 & $54,7 \%$ & 639 & $76,7 \%$ \\
\hline $\begin{array}{l}\text { Interjections/ } \\
\text { onomatopées }\end{array}$ & 150 & $34,0 \%$ & 185 & $22,2 \%$ \\
\hline Majuscules & 50 & $11,3 \%$ & 9 & $1,1 \%$ \\
\hline \hline & 441 & $100 \%$ & 833 & $100 \%$ \\
\hline
\end{tabular}

Tableau 3 - Nombre d'occurrences et pourcentages des procédés expressifs chez les adolescents et les adultes

Chez les adultes, les procédés expressifs constituent près du tiers de tous les procédés scripturaux mis à contribution. Au premier rang, on trouve les caractères écho qui, avec 639 occurrences, totalisent près de $77 \%$ de cette catégorie, par ex. :

- A IV $60<$ Jenniee $>$ tresssssssssssss tresssssssssssssssssss bien.

Viennent au deuxième rang les onomatopées/interjections qui, avec 185 occurrences, comptent pour un peu plus du cinquième des procédés expressifs, par ex. :

- A XIV $36<$ Amy|Ly $>$ berk degeux des frites en se levant.

Signalons qu'un peu plus de la moitié ( $55 \%$ ) des onomatopées/interjections se trouvent en combinaison avec des caractères écho, un procédé expressif en reforçant un autre, par ex. :

- A V $396<$ j4o> Sloupie smackkkkkkkkkkk juste a point avant le caractere me change..hihihi.

Finalement, au troisième rang, on trouve l'emploi de majuscules qui représente un maigre $1,1 \%(9$ occurrences), par ex. :

\section{- A V $117<$ Nicole53> OUPS SCUSE.}

Dans le groupe des jeunes, les procédés expressifs apparaissent dans une proportion moindre, totalisant un peu moins de $15 \%$ de tous les procédés. Comme chez les adultes, les caractères écho dominent clairement et constituent plus de la moitié des procédés expressifs ( 241 occurrences), par ex. :

- J XXII $99<$ _Crazy_> trop jeune de caractereeeeeeeeeeeeeeeeeeeeeee.

Comme chez les adultes, on trouve au deuxième rang les onomatopées/interjections qui comptent pour un peu plus du tiers des procédés expressifs (150 occurrences), par ex. : 
- J III $71<$ AlLySoN $>$ ouin mais tchute la.

La combinaison des onomatopées/interjections avec des caractères écho est cependant plus faiblement présente chez les jeunes - un peu plus du tiers des onomatopées/interjections - que chez les adultes, par ex. :

- J XXII $127<$ Mysterio $>$ Brrrr sois pas si froide allons :|.

Comme chez les adultes, l'emploi de majuscules occupe le troisième rang pour totaliser $11,3 \%(50$ occurrences). Toutefois, la proportion des majuscules est plus grande que dans le groupe des aînés, qui rappelons-le, ne totalisait que $1,1 \%$ des procédés expressifs, par ex. :

- J XXI $87<$ MaGikGiRl> sauf ke c vraiment ÉTRANGE.. ki commence de bonnheure dememe.. meme mon frere ki é o cegep commence juste le 21.

\subsubsection{Les substitutions de graphèmes}

Les résultats pour les substitutions de graphèmes, qui occupent le troisième rang en importance, sont inscrits dans le Tableau 4. Ce procédé se démarque chez les adolescents et totalise 10,9\% (soit 277 occurrences) de l'ensemble des catégories. Chez les adultes, les substitutions de graphèmes sont plus faiblement représentées $(4,4 \%$, soit 97 occurrences).

\begin{tabular}{|c|c|c|c|c|}
\hline \multirow[t]{2}{*}{$\begin{array}{c}\text { Substitutions de } \\
\text { graphèmes }\end{array}$} & \multicolumn{2}{|c|}{$\mathbf{J}$} & \multicolumn{2}{|c|}{$\mathbf{A}$} \\
\hline & $\begin{array}{l}\text { Nb } \\
\text { occ. }\end{array}$ & $\%$ & Nb occ. & $\%$ \\
\hline $\mathrm{c}, \mathrm{c}>\mathrm{s}$ & 185 & $66,8 \%$ & 76 & $78,4 \%$ \\
\hline $\mathbf{c}>\mathbf{k}$ & 54 & $19,5 \%$ & 18 & $18,6 \%$ \\
\hline oi $>$ wa & 18 & $6,5 \%$ & 1 & $1,0 \%$ \\
\hline oi $>$ oua & 9 & $3,3 \%$ & 1 & $1,0 \%$ \\
\hline $\mathbf{s}>\mathbf{z}$ & 7 & $2,5 \%$ & 1 & $1,0 \%$ \\
\hline oi $>$ oa & 4 & $1,4 \%$ & 0 & $0,0 \%$ \\
\hline$\sum$ & 277 & $100 \%$ & 97 & $100 \%$ \\
\hline
\end{tabular}

Tableau 4 - Nombre d'occurrences et pourcentages des substitutions de graphèmes chez les adolescents et les adultes

Dans le groupe des adolescents, la substitution de $\mathbf{s}$ à $\mathbf{c}$, ç est fortement majoritaire $(66,8 \%$, soit 185 occurrences). Le second pourcentage en importance est celui de la substitution de $\mathbf{k}$ à $\mathbf{c}$ (près du cinquième du total des substitutions de graphèmes, soit 54 occurrences). Les autres manipulations, soit les substitutions de wa à oi, de oua à oi, de $\mathbf{z}$ à $\mathbf{s}$ et de oa à oi, constituent au total seulement 13,7\% (soit 38 occurrences).

On observe les mêmes tendances dans le groupe des adultes. La sous-catégorie la plus représentée, et de loin, est celle du passage de c, ç à $\mathbf{s}(78,4 \%$, soit 76 occurrences). Suit la substitution de $\mathbf{k}$ à $\mathbf{c}$ qui totalise près du cinquième du total de l'ensemble, soit 18 occurrences. Les trois dernières souscatégories, les substitutions de wa à oi, de oua à $\mathbf{o i}$, et de $\mathbf{z}$ à $\mathbf{s}$, sont très faiblement représentées $(3,0 \%$, soit 3 occurrences). 


\subsubsection{Les neutralisations en finale absolue}

La quatrième catégorie en importance, les neutralisations en finale absolue, est très faiblement représentée dans nos populations $(6,0 \%$ chez jeunes, soit 153 occurrences vs $4,8 \%$ chez les adultes, soit 104 occurrences). Le Tableau 5 présente la répartition des différentes sous-catégories des neutralisations en finale absolue.

\begin{tabular}{|l||r|r|r|r|}
\hline \multicolumn{1}{|l||}{$\begin{array}{l}\text { Neutralisations en finale } \\
\text { absolue }\end{array}$} & \multicolumn{2}{|c||}{ J } & \multicolumn{2}{c|}{ A } \\
\hline & $\begin{array}{c}\text { Nb } \\
\text { occ. }\end{array}$ & \multicolumn{1}{c|}{$\%$} & Nb occ. & \multicolumn{1}{c|}{$\%$} \\
\hline ai, ais, es, est > é & 94 & $61,4 \%$ & 60 & $57,7 \%$ \\
\hline é > er & 50 & $32,7 \%$ & 36 & $34,6 \%$ \\
\hline er $>$ é & 9 & $5,9 \%$ & 8 & $7,7 \%$ \\
\hline \hline & 153 & $100 \%$ & 104 & $100 \%$ \\
\hline
\end{tabular}

Tableau 5 - Nombre d'occurrences et pourcentages des neutralisations en finale absolue chez les adolescents et les adultes

Dans le groupe des adolescents, le procédé le plus important proportionnellement est la neutralisation des graphèmes complexes ai, ais, es, est en é à la finale absolue. Cette manipulation compte pour un peu plus de $60 \%$ du total des neutralisations en finale absolue, par ex. :

- J II $113<\{\mathrm{M}\{\mathrm{eL}\} \mathrm{i}\}>$ mé <mais> sé <c'est> surment une connerie:P;

- J XIII $52<$ RoXie_> elle veut maigrir dé $<$ des $>$ doigt;

- J XV $176<$ AuDrEeAnN> Jté <je t'ai> déja assez pardonner comme sa seb.

Au deuxième rang, on trouve la neutralisation de la finale é en er (près du tiers du total de l'ensemble des neutralisations en finale absolue), Cette manipulation touche surtout les participes passés et occasionnellement des substantifs, par ex. :

- J VIII $1<$ AbenGaDoN> moé j't'en conger <congé> en fds prochaine, srais popire qu'on fasse queque chose tlm;

- J IX $13<$ judy> Elle est pas arriver <arrivée > chez elle encore...;

Cette substitution de luxe - deux caractères pour un seul - est nettement prépondérante par rapport à la neutralisation de la finale er en é, qui compte pour à peine $6 \%$ de cette catégorie, par ex. :

- J XII 16 <golfvr6> savais tu sa été long une sem sans te parlé <parler> lol;

Chez les adultes, les résultats globaux sont similiaires. Près de $58 \%$ des neutralisations en finale absolue impliquent la neutralisation des graphèmes complexes ai, ais, es, est en é à la finale absolue, par ex. :

- A I $291<$ miNoU^$^{\wedge}>$ sylvy_^$^{\wedge}$ té $<$ t’es $>$ pu la loll111111111111111;

- A V $74<$ Nicole 53> Le-Furtif cé <c'est> bien ca sa te garde jeune bébé.

De la même manière que dans le groupe des jeunes, la neutralisation de la finale é en er occupe le deuxième rang, soit un peu plus du tiers des neutralisations en finale absolue, par ex. :

- A I $133<$ police40> sylvy_^ c tomber <tombé> fort tantot ici;

- A XVI $49<$ ladylight $>$ rock57 désoler < désolée> alors. 
Finalement, la neutralisation de la finale er en é arrive au troisième rang et ne représente que 7,7\% de l'ensemble, par ex. :

- A I $63<$ miNoU^$^{\wedge}>$ sylvy_^$_{-}^{\wedge}$ pi a la fin de sa 2 ieme année elle va pouvoir komencé $<$ commencer $>$ la thanatopraxie;

\section{Conclusion}

Dans cet article, nous avions voulu, d'une part, classifier les usages scripturaux en français en situation de clavardage et d'autre part, vérifier si ces usages sont les mêmes chez les adolescents et les adultes. Les résultats nous montrent que, dans l'ensemble, les deux groupes recourent abondamment à des stratégies d'abréviation et à des moyens propres à reproduire les paramètres de la conversation en face-à-face. Ainsi, les procédés abréviatifs et les procédés expressifs sont-ils les deux catégories dominantes dans nos deux populations. À la lumière de nos résultats, nous constatons que le groupe des adultes, tout en recourant aux différents procédés, est plus réticent à transgresser l'orthographe conventionnelle que ne l'est le groupe des adolescents. Les premiers ont surtout tendance à employer des procédés expressifs, visant à combler l'absence d'informations paralinguistiques ou extralinguistiques en situation de clavardage. Les adolescents, eux, se singularisent par exemple en multipliant les substitutions de graphèmes.

De nombreuses pistes apparaissent à l'issue de ce travail. Nous sommes à cet effet à étendre notre étude à des langues dont le degré de correspondance phonético-graphique diffère. En vertu de l'hypothèse de la profondeur de l'orthographe (orthographic depth hypothesis [ODH]) (Katz et Frost 1992) selon laquelle un système orthographique transparent (comme l'italien, l'espagnol ou le serbo-croate) code les phonèmes directement dans l'orthographe, nous vérifierons si nos résultats pour le français peuvent être généralisés à des langues dont l'orthographe est dite " transparente » (l'espagnol) vs des langues dont l'orthographe est dite "opaque » (l'anglais). À cet égard, il sera intéressant de répertorier les procédés scripturaux des adolescents hispanophones par rapport à ceux des adultes car la marge de transgression orthographique dans cette langue est bien plus étroite que pour le français ou l'anglais. Il s'ensuit que les écarts entre les groupes de jeunes et d'adultes pourraient se manifester différemment.

La question de la qualité de la langue dans les dispositifs de CMO suscite nombre de débats et de controverses. Depuis quelques années, les médias publient des articles portant sur les « détournements orthographiques » chez les utilisateurs de ces nouveaux moyens de communication. Certains des titres sont révélateurs de l'inquiétude de l'opinion publique : «Plus les ados clavardent et plus ils écrivent mal! » (Sébastien Ménard, Journal de Montréal, 12 mai 2005), " Le clavardage, massacre de la langue ou renaissance linguistique ? » (Frédérique Doyon, Le Devoir, 1er février 2007), « L'ordi, ami ou ennemi du français ?» (Violaine Ballivy, La Presse, 9 février 2008), «Les textos dans le collimateur du chef de l'État » (Agnès Leclair, Le Figaro, 15 février 2008). Ces réactions débouchent sur une autre piste de recherche qui consisterait à vérifier si la $\mathrm{CMO}$ est réellement impliquée dans l'affaiblissement du niveau d'orthographe chez les adolescents. Manesse et Cogis (2007) ont démontré qu'il y avait un accroissement des erreurs d'orthographe - et plus spécifiquement pour l'orthographe grammaticale - entre 1987 et 2005 chez les élèves du CM2 à la troisième. Il serait souhaitable de déterminer l'impact de la CMO et des phénomènes linguistiques afférents sur différents types de textes (par ex. productions écrites de niveau secondaire, dictées, etc.), en vérifiant la présence ou l'absence de phénomènes graphiques et scripturaux dans ces textes. Selon David et Goncalves (2007), qui ont fait une réflexion sur l'impact des $\mathrm{SMS}^{7}$ sur la maîtrise de la langue, nous sommes en présence d'une digraphie qui «pourra/pourrait réellement relativiser le poids excessif des normes orthographiques ».

\section{Remerciements}

Je tiens à remercier ma directrice de recherche, Louise Dagenais de l'Université de Montréal, pour son appui et ses interventions tout au long de la réalisation de mon étude. Nous remercions également le Conseil de recherche en sciences humaines du Canada pour son soutien financier (2004-2005). 


\section{Références}

Anis, J. (dir.). (1999). Internet, communication et langue française. Paris : Hermès Science Publications.

Anis, J. (dir.). (2001). Parlez-vous texto ? Guide des nouveaux langages du réseau. Paris : Le Cherche Midi.

Borrell, A. (1986). « Nouveau français », « parler jeune » ou « langue des cités »? Remarques sur un objet linguistique médiatiquement identifié, Langue française, 114, 6-15.

Clark, H. H. \& S. E. Brennan (1991). Grounding in Communication. In L. B. Resnick, J. M. Levine \& S. D. Teasley (eds), Perspectives on Socially Shared Cognition. Washington : American Psychological Association, 127-149.

Crystal, D. (2006). Language and the Internet. Cambridge : Cambridge University Press.

David, J. et H. Goncalves (2007). L'écriture électronique, une menace pour la maîtrise de la langue ? Le Français aujourd'hui, 156, 39-48

Dubois, J. et al. (2001). Dictionnaire de linguistique. Paris : Larousse-Bordas.

Eckert, P. (1988). Adolescent Social Structure and the Spread of Linguistic Change. Language in Society, 17, 183207.

Frei, H. (1929). La grammaire des fautes. Paris : Geuthner.

Herring, S. C. (2000). Linguistic Approaches to Computer-Mediated Communication. Communication présentée à la Jornada sobre Communicació Mediatitzada per Ordinador en Català (Barcelone, 1er décembre 2000).

Jeay, A.-M. (1991). Les messageries télématiques. Paris : Eyrolles.

Katz, L. \& R. Frost (1992). The Reading Process is Different for Different Orthographies: The Orthographic Depth Hypothesis. In L. Katz \& R. Frost (eds), Orthography, Phonology, Morphology and Meaning. Amsterdam : Elsevier North Holland Press, 67-84.

Kiesler, S., Siegel, Jane \& T. W. McGuire (1984). Social Psychological Aspects of Computer-mediated Communication. American Psychologist, 39-10, 11-26.

Labov, T. (1992). Social and Language Boundaries among Adolescents. American Speech, 67-4, 339-366.

Lacerte, C. (2006). Représentations sociales du code conversationnel du clavardage chez les jeunes et chez les experts québécois. Mémoire de maîtrise, Université du Québec à Montréal.

Llisterri, J. (2002). Marcas fonéticas de la oralidad en la lengua de los chats: elisiones y epéntesis consonánticas, Revista de Investigación Lingüistica, 2-5, 61-100.

Luzzati, D. (1991). Oralité et interactivité dans un écrit Minitel. Langue Française, 89, 99-109.

Manesse, D. \& D. Cogis (2007). Orthographe : à qui la faute? Issy-les-Moulineaux : ESF.

Palfreyman, D. \& M. Al Khalil (2003). A Funky Language for Teenzz to Use: Representing Gulf Arabic in Instant Messaging. Journal of Computer-Mediated Communication, 9-1.

Panckhurst, R. (1997). La communication «médiatisée » par ordinateur ou la communication « médiée »par ordinateur? Terminologies nouvelles, 17, 56-58.

Pierozak, I. (2000). Les pratiques discursives des internautes. Le français moderne, 68-1, 109-129.

Sebba, M. (2003). Spelling Rebellion. In J. K. Androutsopoulos \& A. Georgakopoulou (eds), Discourse Constructions of Youth Identities. Amsterdam and Philadelphia : John Benjamins, 151-172.

Tagliamonte, S. \& D. Denis (soumis). LOL for real! Instant Messaging, Teen Language and Linguistic Change. American Speech.

Tatossian, A. et L. Dagenais. À paraître (décembre 2008). Le scripto-clavardage en français : adolescents $v s$ adultes. Cahiers de lexicologie.

Tatossian, A. (2005). L'orthochat du français : adolescents vs adultes. Mémoire de maîtrise, Université de Montréal.

Walter, H. (1984). L'innovation lexicale chez les jeunes Parisiens. La linguistique, 20-2, 69-84. 
Werry, C. C. (1996). Linguistic and Interactional Features of Internet Relay Chat. In S. C. Herring (ed.), ComputerMediated Communication: Linguistic, Social and Cross-Cultural Perspectives. Amsterdam and Philadelphia : John Benjamins, 47-63.

Zelenkauskaite, A. \& S. C. Herring (2006). Gender Encoding of Typographical Elements in Lithuanian and Croatian IRC. In F. Sudweeks \& C. Ess (eds), Actes du colloque Cultural Attitudes Towards Technology and Culture 2006 (Tartu, 28 juin-1e juillet 2006). Murdoch : Murdoch University Press.

\footnotetext{
${ }^{1}$ Mot-valise formé à partir des mots CLAVier et bavARDAGE proposé en octobre 1997 par l'Office québécois de la langue française pour désigner une « activité permettant à un internaute d'avoir une conversation écrite, interactive et en temps réel avec d'autres internautes, par clavier interposé » (Grand dictionnaire terminologique). Le terme de " clavardage » dénomme une pratique appelée chat en France.

${ }^{2}$ Certains logiciels de messagerie instantanée (par exemple MSN Messenger, Skype, Google Talk) permettent à la fois de clavarder et de communiquer en mode audio et/ou vidéo avec un microphone et une caméra Web (webcam). Ce type de CMO peut donner l'impression d'une rencontre en face-à-face entre utilisateurs.

${ }^{3}$ Un message désigne une intervention qu'envoie un clavardeur dans un canal (apparaissant après le pseudonyme). Par ex., la séquence en gras représente un message : $<\mathrm{Ro} 0 \mathrm{oXxXy}>$ ya kek1 ki sé c koi le chan de jeuquizz.

${ }^{4}$ Le choix du réseau Undernet repose sur les critères suivants : 1) présence d'une population conséquente ; 2) présence de nombreux canaux francophones stables.

${ }^{5}$ En tenant compte du nom des divers canaux, par ex. \#12-19 vs \#40ans\&+, et des propos qui y sont tenus, on peut évaluer avec une certaine assurance les populations adulte vs jeune qui s'y expriment. Mais bien entendu, l'anonymat des participants reste un écueil en situation de clavardage. Concernant les limites de l'anonymat, voir l'étude de Zelenkauskaite et Herring (2006) qui traite des différences inter sexes (gender studies) des caractéristiques orthographiques et typographiques du lituanien et du serbo-croate sur IRC.

${ }^{6} V S$ acronymie.

${ }^{7}$ Les SMS (Short Message Service) réfèrent au service proposé conjointement à la téléphonie mobile permettant de transmettre des messages écrits de taille restreinte ( 160 caractères pour les alphabets latins, et 70 caractères pour les alphabets non latins tels que l'arabe, le chinois, le coréen, le japonais, le russe). Pour désigner ce type de message, on emploie aussi le terme « texto ».
} 